\title{
Organised polarisation variability in radio pulsars and consequences for emission theory
}

\author{
Cristina-Diana Ilie ${ }^{1}$ and Patrick Weltevrede ${ }^{1}$ \\ ${ }^{1}$ Jodrell Bank Centre of Astrophysics, University of Manchester, \\ Alan Turing Building, M13 9PL, Manchester, United Kingdom \\ email: cristina.ilie@postgrad.manchester.ac.uk
}

\begin{abstract}
The aim of this work is to explore the connection between variability in single pulse intensity and periodic switching of the position angle (PA) of the linear polarisation and how this relates to the radio emission mechanism. There are five pulsars reported in the literature for which the PA is seen to periodically change in tandem with the variability in their pulse shapes. This behaviour is seemingly incompatible with two well established models of the radio emission mechanism. The purpose of this study is to investigate in a systematic way whether this phenomenon is common or if only happens in special cases, using a high-quality sample of pulsar data observed with the Parkes telescope. We show that the connection between polarisation variability and intensity variability is more common than previously expected.
\end{abstract}

Keywords. pulsars : general, polarization, PSR B0031-07

\section{Introduction}

Since the discovery of pulsars, 50 years ago (Hewish et al. 1968), a lot of progress has been made in understanding how the radio emission mechanism of pulsars works, however a theoretical model of this mechanism that can explain all the observations has not yet been developed. The aim of this work is to explore the connection between variability in single pulses intensity and periodic switching of the position angle (PA) of the linear polarisation and how this relates to the radio emission mechanism. There are five pulsars reported in the literature for which the PA is seen to periodically change in tandem with the variability in their pulse shapes (Ramachandran et al. 2002, Rankin \& Ramachandran 2003, Edwards 2004). This behavior is seemingly incompatible with two well established models of the radio emission mechanism, which will be further discussed.

The first model aims to explain the periodic modulation observed in the shape of individual pulses in at least a third of the pulsar population (Weltevrede et al. 2006). Individual pulses display very complex behaviour and have been observed to systematically shift in time forming diagonal intensity bands in the pulse-stack (left panel of Fig. 1), a phenomenon referred to as drifting subpulses (Drake \& Craft 1969). An important periodicity to define is $P_{3}$ : the vertical spacing between the diagonal driftbands, which represents the periodicity with which the drifting subpulse pattern repeats. $P_{3}$ is normally defined in terms of the pulse period, $P$. As drifting is so common in pulsars, it is believed to be an integral part of the emission mechanism. One of the most notable models aiming to explain this is referred to as the carousel model and suggests that the emission beam is made out of subbeams of emission circulating around the magnetic pole (Ruderman \& Sutherland 1975). Each subpulse is thought to originate from a subbeam in the carousel. As the carousel rotates, the subbeam will gradually move across the line of sight, hence a systematic shift in time will occur in the pulse-stack. 

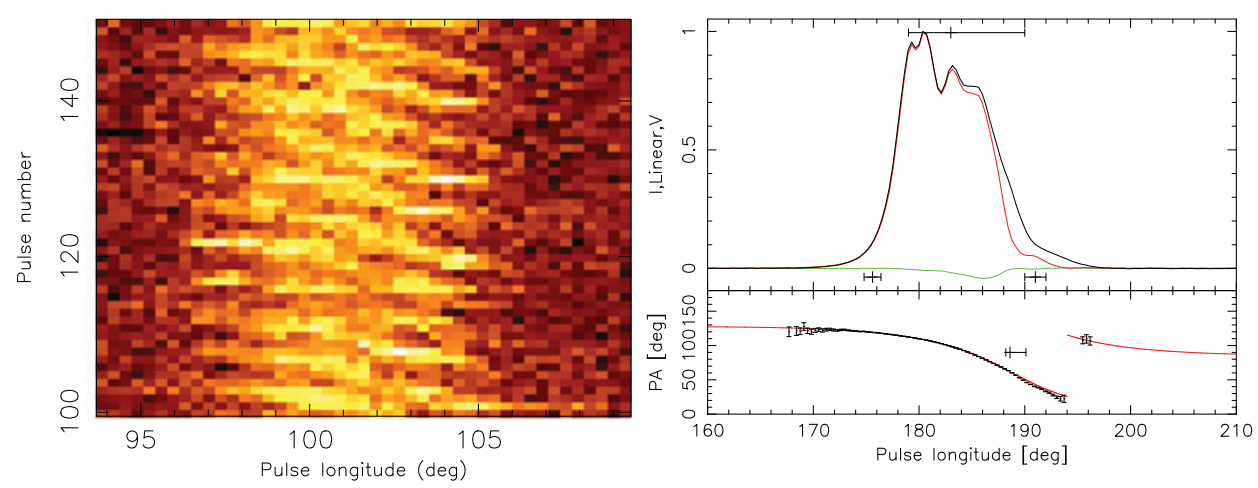

Figure 1. Left: A sequence of 50 individual pulses from the intensity pulse-stack of PSR B0818-13 displaying drifting subpulses with $P_{3}=5 P$. Right: PSR J0742-2822 (Figure taken from Rookyard et al. (2015)). The polarimetric integrated pulse profile is displayed in the top panel. The PA swing is plotted in the bottom panel as a function of rotational phase. The PA points are only displayed when the significance of the linear polarisation exceeds 3 sigma.

The second model explains the switching of the PA of the linear polarisation between two states, referred to as Orthogonally Polarised Modes (OPMs). The PA curve is determined by the projection of the dipolar magnetic field lines on the plane of the sky, tracing a typical S-swing change during a stellar rotation, as explained by the Rotating Vector Model (RVM) (Radhakrishnan \& Cooke 1969). The right-hand panel of Fig. 1 shows an example of polarimetric pulse profile, as well as the average PA as a function of pulse longitude. The curve in the lower panel represents the fitted RVM model to the average PA (Rookyard et al. 2015). As seen in Fig. 1 around pulse longitude $194^{\circ}, 90^{\circ}$ discontinuities can occur in the PA swing. The RVM model has been modified in order to explain these with the co-existence of two OPMs of radiation (Backer et al. 1976). If two adjacent field lines are dominated by different OPMs then a rapid $90^{\circ}$ jump is expected to occur in the PA curve.

The origin of the alternating dominance of OPMs is still uncertain. Some authors (e.g. Gangadhara 1997) believe the production of two orthogonally polarised modes is intrinsic to the radio emission mechanism, however using this theory it is hard to explain partially polarised modes observed in some pulsars. Other authors (e.g. Melrose 1979, Arons \& Barnard 1986) suggest that the two OPMs are two natural modes of propagation in a cold highly-relativistic magnetised plasma, the Ordinary mode (O mode) and the eXtraodinary mode ( $\mathrm{X}$ mode). The $\mathrm{X}$ mode propagates without being affected by refraction, thus its trajectory is almost a straight line from the emitting region. The $\mathrm{O}$ mode propagates on a curved path before detaching.

From the theory of the carousel model and OPMs, we would not immediately expect a connection between the two models. In the RVM model, the PA depends on the projection of the magnetic field lines which do not change over time, hence we would not expect that the switching between two PA states would be related to variations on short timescales in the plasma, as in the case of drifting subpulses. Ramachandran et al. (2002) showed that for PSR B0809+74 the switches between the two OPMs at a single pulse level were modulated by the the frequency of drifting subpulses. This behaviour is seemingly incompatible with the two well established models discussed. Studies of this phenomena are rare and have not been done in a systematic way, hence the aim of this work is to investigate how common is this effect. A sample of 34 pulsars which was selected based on the fact that all these sources show evidence for different OPMs dominating at different rotational phases, indicating that both OPMs are active in these pulsars. This information 

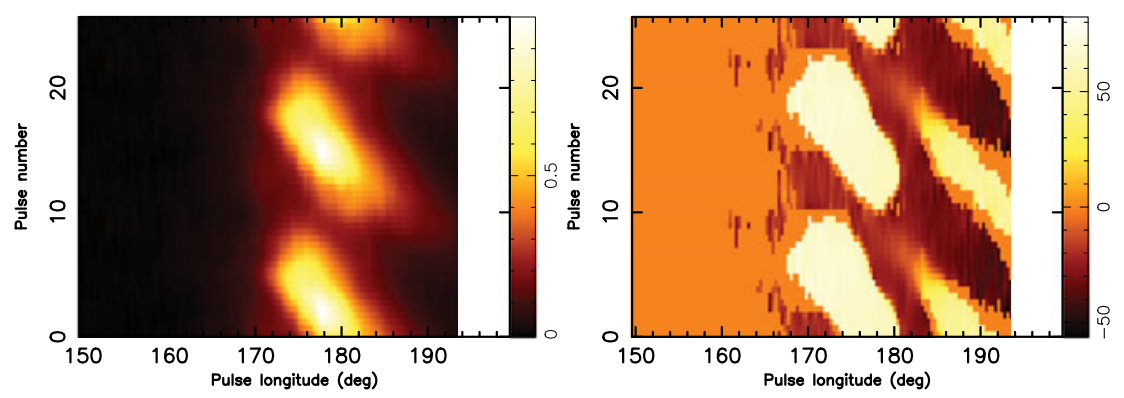

Figure 2. PSR B0031-07 Mode A of drifting. Left: $P_{3}$-fold of the total intensity. Right: $P_{3}-$ fold showing the PA. In both plots, the average driftband was displayed twice for continuity.

was revealed utilising published time-averaged data preventing pulse-shape variability to be quantified. Furthermore, for all the sources drifting subpulses were identified from either the literature or archival data without polarisation information. The single-pulse polarisation data was collected using the Parkes Telescope at a frequency of $1369 \mathrm{MHz}$ and $256 \mathrm{MHz}$ bandwidth.

\section{Results}

In order to look at the PA "flickering" at a single pulse level (between the two different OPM states), even in pulsars with low degree of polarisation, we produced the average driftband using a technique called $P_{3}$-folding. The principle of this method is that the data is averaged over the modulation cycle in order to obtain the average shape of a driftband. However, there is a problem when averaging successive drift bands over large stretches of data. This is because the value of $P_{3}$ is not fixed and changes within an interval of values. Hence if folding is performed without taking into account these variations issues can arise (e.g. Deshpande \& Rankin 2001, van Leeuwen et al. 2002). Hassall et al. (2013) were the first to take into account these variations using the PSRSALSA software package and this is the tool which was used to analyse our pulsars (Weltevrede 2016).

One of the pulsars from our sample was B0031-07. This pulsar is known to exhibit drifting subpulses with three different stable drift modes, $P_{3}=13 P$ (mode A), $P_{3}=7 P$ (mode B) and $P_{3}=4 P$ (mode C) (Huguenin et al. 1970). However, it has been observed that the occurrence of these drift modes depends on observing frequency (Smits et al. 2005, 2007), with only mode A and mode B being visible at higher frequencies. At our observing frequency, we indeed identified these two modes as well. A link between the polarisation properties and the drifting subpulses for PSR B0031-07 for both mode A and mode B was previously found by Chen (2015), however the analysis was hampered by strong instrumental intensity variations on a timescale comparable with the rotation period of the neutron star. The $P_{3}$-folds of the intensity and PA pulse-stacks for the mode A of drifting data are displayed in Fig. 2.

When looking at the $P_{3}$-fold representing the PA pulse-stack (right of Fig. 2), we can clearly see the two OPMs (the lighter region and the darker region, white and brown in the online version) periodically switching, and this change is modulated by $P_{3}$. Comparing this plot with the $P_{3}$-fold of the intensity pulse-stack (left of Fig. 2), it is interesting to note that the white OPM occurs at the same phases as the intensity driftband, while the brown OPM occurs in between the intensity driftbands, at pulse longitudes of $170^{\circ}-182^{\circ}$. Past these pulse longitudes, the intensity is lower and it appears to trace the brown OPM. 
These and similar results will be described in more detail in a future publication (Ilie et al. in prep).

Out of the 34 pulsars which were observed in this study, we identified 14 which had both OPMs dominating at different times at the same pulse longitudes (i.e. OPM flickering). Out of these 14 pulsars, 6 displayed drifting subpulses and a connection between $P_{3}$ and the switching of OPMs was established. For the rest 8 pulsars, a connection between intensity and polarisation was established but a direct link to a $P_{3}$ value could not be established. These initial results let us to believe that in general, OPM flickering is related to variability in total intensity, which presumably can be traced back to changes in the electron-positron acceleration region close to the neutron star surface.

\section{Revisiting the carousel model}

The connection between polarisation properties and the periodicity of drifting subpulses must be a more general property of the emission mechanism than initially believed. Rankin \& Ramachandran (2003) suggested that in order to explain these observations, there should be two carousels, one for each OPM, offset from each other both in the radial direction as well as in the azimuthal direction. A possible explanation for this would be using the natural modes in a plasma, the $\mathrm{O}$ and $\mathrm{X}$ modes. As discussed before, one of the modes is more affected by refraction, thus these two carousels could be images of one carousel. A more detailed analysis of our data will reveal if this picture in general is supported.

\section{Acknowledgments}

This publication has received funding from the European Union's Horizon 2020 research and innovation programme under grant agreement No 730562 [RadioNet]

\section{References}

Arons, J. \& Barnard, J. J. 1986, ApJ, 302, 120

Backer, D. C., Rankin, J. M., \& Campbell, D. B. 1976, Nature, 263, 202

Chen, T. 2015, MSc Thesis, The University of Manchester

Deshpande, A. A. \& Rankin, J. M. 1969, MNRAS, 322, 438

Drake, F. D. \& Craft, H. D. 1969, Nature, 220, 231

Edwards, R. T. 2004, A\&A, 426, 677

Gangadhara, R. T. 1997, A\&A, 327, 155

Hassall, T. E., Stappers, B. W., Weltevrede, P. et al. 2013, A\&\&A, 552, A61

Hewish, A., Bell, S. J., Pilkington, J. D. H., Scott, P. F., \& Collins, R. A. 1968, Nature, 217, 709

Huguenin, G. R., Taylor, J. H., \& Troland, T. H. 1968, ApJ, 162, 727

Ilie, C.D., Weltevrede, P. \& Johnston, S. in prep,

Melrose, D. B. 1979, PASA, 32, 61

Radhakrishnan, V. \& Cooke, D. J. 1969, Ap. Lett., 3, 225

Rankin, J. M. \& Ramachandran, R. 2003, ApJ, 590, 411

Ramachandran, R., Rankin, J. M., \& Stappers, B. W. et al. 2002, A\&SA, 381, 993

Rookyard, S. C.,Weltevrede, P., \& Johnston, S. 2015, MNRAS, 446, 3367

Ruderman, M. A. \& Sutherland, P. G. 1975, ApJ, 196, 51

Smits, J. M., Mitra, D., \& Kuijpers, J. 2005, A $\mathscr{J} A$, 440, 683

Smits, J. M., Mitra, D., \& Stappers, B. W. et al. 2007, A\& $A, 465,575$

van Leeuwen, A. G. J., Kouwenhoven, M. L. A., \& Ramachandran, R. et al. 2002, A\&A, 387, 169

Weltevrede, P., Edwards, R. T., \& Stappers, B. W. 2006, A $₫ A, 445,243$

Weltevrede, P. 2016, A\& A, 590, 109 